\title{
A GENERAL HOPF LEMMA AND PROPER HOLOMORPHIC MAPPINGS BETWEEN CONVEX DOMAINS IN $\mathbb{C}^{n}$
}

\author{
PETER R. MERCER
}

(Communicated by Clifford J. Earle, Jr.)

\begin{abstract}
We use a general version of the well-known Hopf lemma to study boundary regularity of proper holomorphic mappings between some bounded convex domains in $\mathbb{C}^{n}$ which carry no boundary regularity assumption.
\end{abstract}

\section{INTRODUCTION}

Let $\Omega_{1}$ and $\Omega_{2}$ be domains in $\mathbb{C}^{n}$ and $\mathbb{C}^{m}$ respectively. A continuous mapping $f: \Omega_{1} \rightarrow \Omega_{2}$ is proper provided $f^{-1}(K)$ is compact in $\Omega_{1}$ whenever $K$ is compact in $\Omega_{2}$. If $\Omega_{1}$ and $\Omega_{2}$ are bounded, this is equivalent to the requirement that $f\left(z_{j}\right) \rightarrow \partial \Omega_{2}$ whenever $\left\{z_{j}\right\} \subset \Omega_{1}$ is such that $z_{j} \rightarrow \partial \Omega_{1}$. A biholomorphic mapping is proper since in this case $f^{-1}$ is continuous.

The problem of boundary regularity of proper holomorphic mappings has been studied by many authors (see the survey article $[F]$ and the references therein). In most cases the domains in question are assumed to possess at least $C^{2}$ boundary regularity. This paper studies the problem for certain bounded domains in $\mathbb{C}^{n}$ which carry no such assumption.

In $\S 1$ we fix notation and recall some fundamental ideas, including a generalization of the well-known Hopf lemma which requires only a cone condition on the domain in question rather than boundary smoothness. In $\S 2$ we apply this result to obtain some sufficient conditions on bounded domains $\Omega_{1}, \Omega_{2} \subset \mathbb{C}^{n}$ for a proper holomorphic mapping $f: \Omega_{1} \rightarrow \Omega_{2}$ to have a Hölder continuous extension to $\bar{\Omega}_{1}$. In particular, we study a case where $\Omega_{1}$ and $\Omega_{2}$ are convex with no presupposed boundary regularity.

The content of this paper will constitute part of the author's doctoral thesis. The author is grateful to Ian Graham, his thesis advisor, for numerous useful discussions and suggestions.

\section{Preliminaries}

We recall some important notions to be used in the sequel. $\Omega$ denotes a domain (= connected open set) and $B_{n}$ denotes the unit ball in $\mathbb{C}^{n}$ defined

Received by the editors August 27, 1991 and, in revised form, February 28, 1992.

1991 Mathematics Subject Classification. Primary 32H35; Secondary 32F05. 
via the usual (Hermitian) inner product. If $n=1$, write $B_{n}=\Delta$, the unit disk in $\mathbb{C}$.

Definition 1.1. Let $\Omega \subset \mathbb{C}^{n}$. The Kobayashi metric $\kappa_{\Omega}: T(\Omega) \rightarrow \mathbb{R}^{+}$is given by

$$
\kappa_{\Omega}(z ; v)=\inf \left\{|u|: \exists f \in \operatorname{Hol}(\Delta, \Omega) \text { such that } f(0)=z, f^{\prime}(0) u=v\right\} .
$$

General properties of $\kappa_{\Omega}$ may be found, for example, in [K] or [Kr2].

If $\Omega \Subset \mathbb{C}^{n}$ is convex, $z \in \Omega$, and $v \in \mathbb{C}^{n}$, denote by $r_{\Omega}(z ; v)$ the radius of the largest one complex-dimensional closed disk, centred at $z$, tangent to $v$, and contained in $\bar{\Omega}$. In this case, Graham [G2, G3] showed that for any $v \in \mathbb{C}^{n}$ we have

$$
\frac{|v|}{2 r_{\Omega}(z ; v)} \leq \kappa_{\Omega}(z ; v) \leq \frac{|v|}{r_{\Omega}(z ; v)} \quad \forall z \in \Omega
$$

Let $\Omega \subset \mathbb{C}^{n}$. Recall that an upper semicontinuous function $\varphi: \Omega \rightarrow$ $[-\infty, \infty)$ is plurisubharmonic (plush) if for every $z, w \in \mathbb{C}^{n}$, the function $\lambda \rightarrow$ $\varphi(\lambda z+w)$ is subharmonic on $\Omega_{z w}=\{\lambda \in \mathbb{C}: \lambda z+w \in \Omega\}$. A pluripolar set is the $-\infty$ set of a nontrivial plush function.

We state a theorem, which gathers several important results about proper holomorphic mappings. These results appear in [Ru, Chapter 15] and we adopt the notation used there. Let $\Omega_{1}, \Omega_{2} \subset \mathbb{C}^{n}$, and let $f: \Omega_{1} \rightarrow \Omega_{2}$ be proper holomorphic. Let $E=\left\{z \in \Omega\right.$ : $\left.\operatorname{det}\left[f^{\prime}(z)\right]=0\right\}$. For any set $A$, denote by $\#(A)$ the cardinality of $A$. In this situation we have

Theorem 1.2. (i) $f\left(\Omega_{1}\right)=\Omega_{2}$.

(ii) $\Omega_{2} \backslash f(E)$ is open, connected, and dense in $\Omega_{2}$.

(iii) $f(E)$ is an analytic subvariety of $\Omega_{2}$.

(iv) There is a positive integer $m$ (called the multiplicity of $f$ ) such that:

(a) If $w \in f(E)$ then $\#\left(f^{-1}(w)\right)<m$.

(b) If $w \in \Omega_{2} \backslash f(E)$ then $\#\left(f^{-1}(w)\right)=m$ and

there is a neighbourhood $W$ of $w$ and $m$ disjoint open connected sets $U_{1}, \ldots$, $U_{m} \subset \Omega_{1}$ such that $f^{-1}(W)=U_{1} \cup \cdots \cup U_{m}$ and $f_{j}=\left.f\right|_{U_{j}}$ is biholomorphic on $U_{j}$ with $f \circ f_{j}^{-1}(w)=w, 1 \leq j \leq m$.

We fix some further notation. For $z \in \Omega \subset \mathbb{C}^{n}$, denote by $d_{\Omega}(z)$ the Euclidean distance from $z$ to $\partial \Omega$. For $p \in \mathbb{C}^{n}, \theta \in(0, \pi), v \in \partial B_{n}$ (considered as a unit vector), and $r>0$, denote by $\Gamma(p, \theta, v, r)$ the open cone in $\mathbb{C}^{n}$ with vertex $p$, aperture $\theta$, axis along $v$, and height $r$. To be explicit, set $H=\left\{z \in \mathbb{C}^{n}: \operatorname{Re}\langle z, v\rangle=0\right\} ; H$ is the $(2 n-1)$ real-dimensional boundary of a half space $\Pi$, which has $v$ as an inner unit normal vector. Thus

$$
\Gamma(p, \theta, v, r)=\left\{z \in \Pi+p:|z-p|<a d_{\Pi+p}(z),|z-p|<r\right\},
$$

where $a>1$ is given by $\theta=2 \cos ^{-1}(1 / a)$. The axis of $\Gamma(p, \theta, v, r)$ is the segment $\{p+t v: 0<t<r\}$.

Definition 1.3. Let $\Omega \subset \mathbb{C}^{n}$ and let $\theta \in(0, \pi)$. We say that $\Omega$ satisfies a $\theta$-cone condition if there is an $r>0$ with the following property: Each $z \in \Omega$ sufficiently close to $\partial \Omega$ lies on the axis of a cone $\Gamma(p, \theta, v, r) \subset \Omega$ for some $p \in \partial \Omega, v \in \partial B_{n}$. 
Such a condition arises in potential theory and the theory of partial differential equations. For example, a Lipschitz domain (a domain whose interior and boundary are given locally by a Lipschitz function) satisfies a $\theta$-cone condition.

The following is the promised version of the Hopf lemma, the proof of which is a modification of that of [FS, Proposition 12.2]. We are grateful to the referee for bringing to our attention that an even more general version is known $[\mathrm{O}$, Mi1, Mi2].

Proposition 1.4. Let $\Omega \Subset \mathbb{C}^{n}$ satisfy a $\theta$-cone condition. Let $\varphi: \Omega \rightarrow[-\infty, 0)$ be plush. There is $a c>0$ and an $\alpha>1 \quad(\alpha=\pi / \theta)$ such that

$$
\varphi(z) \leq-c d_{\Omega}^{\alpha}(z) \quad \forall z \in \Omega .
$$

Remark 1.4.1. If $\Omega \Subset \mathbb{C}^{n}$ is convex then $\Omega$ satisfies a $\theta$-cone condition (see the proof of Lemma 2.2). The integrated form $k_{\Omega}$ of $\kappa_{\Omega}$ is the well-known Kobayashi distance on $\Omega$ [Roy] (see also [K, Kr2]). We remark further that in this case Lempert [L] showed that for each fixed $z_{0} \in \Omega$ the function $\log \tanh k_{\Omega}\left(z_{0}, \cdot\right)$ is plush on $\Omega$. Now whenever $\varepsilon>0$ is small, we have $-x<\log (1-(1-\varepsilon) x)$ for small $x>0$. Proposition 1.4 together with Lempert's result implies then that there is a $c>0$ (depending only on $z_{0}$ ) and an $\alpha>1$ such that

$$
k_{\Omega}\left(z_{0}, z\right) \leq c-\frac{1}{2} \log d_{\Omega}^{\alpha}(z) \quad \forall z \in \Omega .
$$

This inequality appears in [Me].

Remark 1.4.2. If $\Omega \Subset \mathbb{C}^{n}$ has piecewise smooth boundary in the sense of [R1] then $\Omega$ satisfies a $\theta$-cone condition. Clearly, such a domain need not be convex. Conversely, a (bounded) convex domain need not have piecewise smooth boundary. See also Remark 2.6.1.

\section{APPLICATION TO PROPER HOLOMORPHIC MAPPINGS}

Definition 2.1. Let $\Omega \Subset \mathbb{C}^{n}$ be starshaped with respect to $0 \in \Omega$. The Minkowski Functional $\mu_{\Omega}: \mathbb{C}^{n} \rightarrow \mathbb{R}$ for $\Omega$ with respect to 0 is given by

$$
\mu_{\Omega}(z)= \begin{cases}\inf [t>0: z / t \in \Omega], & z \neq 0, \\ 0, & z=0 .\end{cases}
$$

Then we have $\Omega=\left\{\mu_{\Omega}<1\right\}, \partial \Omega=\left\{\mu_{\Omega}=1\right\}$, and $\Omega^{c}=\left\{\mu_{\Omega}>1\right\}$.

Lemma 2.2. Let $\Omega \Subset \mathbb{C}^{n}$ be convex with $0 \in \Omega$. The function $\mu_{\Omega}-1: \Omega \rightarrow$ $[-1,0)$ is plush and there is a $c>0$ such that

$$
-c d_{\Omega}(z) \leq \mu_{\Omega}(z)-1 \quad \forall z \in \Omega .
$$

Proof. Since $\Omega$ is convex, $\mu_{\Omega}$ is a convex function and the first assertion follows.

There is a $\theta \in(0, \pi)$ and an $r>0$ such that for each $p \in \partial \Omega$ we have $\Gamma_{p}=\Gamma(p, \theta,-p /|p|, r) \subset \Omega$. Now to prove the second assertion it suffices to consider points $z \in \Omega$ near $\partial \Omega$. For such a $z$, set $p=p(z)=z / \mu_{\Omega}(z) \in \partial \Omega$. We may assume that $z \in \Gamma_{p}$. Let $a=\inf [|p|: p \in \partial \Omega]$. Then

$$
\begin{aligned}
d_{\Omega}(z) & \geq d_{\Gamma_{p}}(z)=|p-z| \sin (\theta / 2) \\
& =\left(1-\mu_{\Omega}(z)\right)|p| \sin (\theta / 2) \geq\left(1-\mu_{\Omega}(z)\right) a \sin (\theta / 2),
\end{aligned}
$$

and the result follows. 
Proposition 2.3. Let $\Omega_{1}, \Omega_{2} \Subset \mathbb{C}^{n}$ be convex. Let $f: \Omega_{1} \rightarrow \Omega_{2}$ be proper holomorphic. There is an $\alpha>1$ and constants $a_{1}, a_{2}>0$ such that

$$
a_{1} d_{\Omega_{1}}^{\alpha}(z) \leq d_{\Omega_{2}}(f(z)) \leq a_{2} d_{\Omega_{1}}^{1 / \alpha}(z) \quad \forall z \in \Omega_{1} .
$$

Proof. We may assume that $0 \in \Omega_{2}$; set $\varphi_{2}=\mu_{\Omega_{2}}-1 . f$ is holomorphic and $\varphi_{2}$ is plush (Lemma 2.2), so $\varphi_{2} \circ f$ is plush on $\Omega_{1}$. Now $\Omega_{1}$ satisfies a $\theta$-cone condition, so Proposition 1.4 provides a $\gamma>1$ and a $c>0$ such that

$$
\varphi_{2} \circ f(z) \leq-c d_{\Omega_{1}}^{\gamma}(z) \quad \forall z \in \Omega_{1} .
$$

The left-hand inequality in (2) now follows from Lemma 2.2.

To prove the right-hand inequality we adopt the terminology of Theorem 1.2 and employ some ideas appearing in [P]. We may assume that $0 \in \Omega_{1}$; set $\varphi_{1}=\mu_{\Omega_{1}}-1$. Fix a point $w_{0} \in \Omega_{2} \backslash f(E)$ and let $W$ be a neighbourhood of $w_{0}$ as in Theorem 1.2(iv)(b). Define $\psi_{j}: W \rightarrow U_{j}$ by

$$
\psi_{j}(w)=\varphi_{1} \circ f_{j}^{-1}(w), \quad 1 \leq j \leq m .
$$

The function $\psi(w)=\max \left[\psi_{j}(w): 1 \leq j \leq m\right]$ is then well defined, plush on $\Omega_{2} \backslash f(E)$, and also bounded there. Now by Theorem 1.2(iii), $f(E)$ is an analytic subvariety of $\Omega_{2}$ and as such it is a pluripolar set. The appropriate extension theorem (e.g., [LG, Proposition I.22]) shows that $\psi$ extends to a plush bounded function on all of $\Omega_{2}$, which we denote again by $\psi$.

By Proposition 1.4 there is a $\beta>1$ and a $c_{1}>0$ such that

$$
\psi(w) \leq-c_{1} d_{\Omega_{2}}^{\beta}(w) \quad \forall w \in \Omega_{2} ;
$$

thus,

$$
\psi_{j}(w) \leq-c_{1} d_{\Omega_{2}}^{\beta}(w) \quad \forall w \in \Omega_{2} \backslash f(E), \quad 1 \leq j \leq m .
$$

By Lemma 2.2 there is a $c_{2}>0$ such that

$$
-c_{2} d_{\Omega_{1}}(z) \leq \varphi_{1}(z) \quad \forall z \in \Omega_{1} ;
$$

thus (observing Theorem 1.2(i)),

$$
-c_{2} d_{\Omega_{1}}\left(f_{j}^{-1}(w)\right) \leq \varphi_{1} \circ f_{j}^{-1}(w) \quad \forall w \in \Omega_{2} \backslash f(E), \quad 1 \leq j \leq m .
$$

With $w=f(z),(3)-(5)$ provide a $c_{3}>0$ such that

$$
d_{\Omega_{2}}^{\beta}(f(z)) \leq c_{3} d_{\Omega_{1}}\left(f_{j}^{-1} \circ f(z)\right) \quad \forall z \in \Omega_{1} \backslash E, \quad 1 \leq j \leq m .
$$

Choosing the correct $j$, we have

$$
d_{\Omega_{2}}^{\beta}(f(z)) \leq c_{3} d_{\Omega_{1}}(z) \quad \forall z \in \Omega_{1} \backslash E .
$$

Finally, by continuity and Theorem 1.2(ii), (6) holds for all $z \in \Omega_{1}$. Thus the right-hand inequality in (2) holds, and the proof is complete upon letting $\alpha=\max (\gamma, \beta)$.

Definition 2.4. Let $\Omega \Subset \mathbb{C}^{n}$ be convex. We say that $\Omega$ is $m$-convex if there is an $m \in(0, \infty)$ and a $c>0$ such that for every $v \in \mathbb{C}^{n}$ we have

$$
r_{\Omega}(z ; v) \leq c d_{\Omega}^{1 / m}(z) \quad \forall z \in \Omega .
$$

We remark that a $C^{2}$-bounded domain with positive definite real Hessian is 2-convex. In general (for $n \geq 2$ ) we must have $m \geq 2$. $m$-convex domains are 
the focus of much of [Me]. Consideration of (1) shows that if $\Omega$ is $m$-convex then there is a $c>0$ such that

$$
\frac{|v|}{c d_{\Omega}^{1 / m}(z)} \leq \kappa_{\Omega}(z ; v) \leq \frac{|v|}{d_{\Omega}(z)} \quad \forall(z, v) \in T(\Omega) .
$$

Lemma 2.5. Let $\Omega \Subset \mathbb{C}^{n}$ be convex. Let $\beta \in(0,1)$ and $f \in C^{1}(\Omega)$. Suppose that there is a $c>0$ such that $|\nabla f(z)| \leq c d_{\Omega}^{\beta-1}(z) \forall z \in \Omega$. There is a $c_{1}>0$ such that

$$
|f(z)-f(w)| \leq c_{1}|z-w|^{\beta} \quad \forall z, w \in \Omega .
$$

As such, $f$ extends to a continuous function on $\bar{\Omega}$ and (8) holds there also (i.e., the extension is Hölder continuous with exponent $\beta)$.

Proof. The first assertion follows from appropriate modifications of standard techniques that appear, for example, in [ $\mathrm{Krl}$, Lemma 4.7]. In that lemma $\Omega$ has $C^{2}$ boundary only; the absence of such an assumption in the present case is made up for by the convexity hypothesis. The rest of the lemma follows from elementary arguments.

Proposition 2.6. Let $\Omega_{1}, \Omega_{2} \Subset \mathbb{C}^{n}$ with $\Omega_{1}$ convex and $\Omega_{2}$ m-convex. Let $f: \Omega_{1} \rightarrow \Omega_{2}$ be proper holomorphic. Then $f$ extends to a Hölder continuous mapping on $\bar{\Omega}_{1}$.

Proof. By the distance decreasing property of $\kappa_{\Omega},(7)$, and Proposition 2.3 there is a $c>0$ and an $\alpha>1$ such that

$$
\left|f^{\prime}(z) v\right| \leq \frac{c d_{\Omega_{2}}^{1 / m}(f(z))|v|}{d_{\Omega_{1}}(z)} \leq c d_{\Omega_{1}}^{1 / \alpha m-1}(z)|v| \quad \forall(z, v) \in T(\Omega) .
$$

Thus each component of $f$ satisfies the hypothesis of Lemma 2.5 with $\beta=$ $1 / \alpha m$, and we are done.

Remark 2.6.1. [R1] (respectively [R2]) contains results analogous to Propositions 2.3 and 2.6 in case $\Omega_{1}$ and $\Omega_{2}$ are bounded domains with piecewise smooth strictly pseudoconvex boundaries (respectively, bounded convex domains with real analytic boundaries) and $f: \Omega_{1} \rightarrow \Omega_{2}$ is biholomorphic rather than just proper holomorphic. Berteloot [B] has independently studied Hölder continuity for proper holomorphic maps between certain pseudoconvex domains with piecewise smooth boundaries. See also Remark 1.4.2.

We have already noted that Lemma 2.5 holds if $\Omega \Subset \mathbb{C}^{n}$ is $C^{2}$-bounded rather than convex [Krl, Lemma 4.7]. Also, estimates such as (2) and (7) are already known to hold in situations where the domains in question have good boundary regularity. For example, (2) holds if $\Omega_{1}, \Omega_{2} \Subset \mathbb{C}^{n}$ are $C^{\infty}$. pseudoconvex [R2], or if $\Omega_{1}, \Omega_{2} \Subset \mathbb{C}^{n}$ are $C^{2}$-strictly pseudoconvex; here $\alpha=1[\mathrm{P}]$. Estimate (7) holds if $\Omega \Subset \mathbb{C}^{n}$ is pseudoconvex with real analytic boundary [DF], or if $\Omega \Subset \mathbb{C}^{n}$ is $C^{2}$-strictly pseudoconvex; here $m=2$ [G1] (see also [H]). Consequently, results analogous to Proposition 2.6 hold for such cases $[R 2, P, H, D F]$. Finally, assumptions on $\Omega_{1}$ and $\Omega_{2}$ may be varied considerably among these cases to obtain still more versions of Proposition 2.6. 


\section{REFERENCES}

[B] F. Berteloot, Hölder continuity of proper holomorphic mappings, Studia Math. 100 (1991), 229-235.

[DF] K. Diederich and J. E. Fornaess, Proper holomorphic maps onto pseudoconvex domains with real analytic boundary, Ann. of Math. (2) 110 (1979), 575-592.

[FS] J. E. Fornaess and B. Stensones, Lectures on counterexamples in several complex variables, Math. Notes, vol. 33, Princeton Univ. Press, Princeton, NJ, 1987.

[F] F. Forstnerič, Proper holomorphic mappings: a survey, Preprint Series, Dept. Math. University E. K. Ljubljana 27 (1989), 5-47.

[G1] I. Graham, Boundary regularity of the Caratheodory and Kobayashi metrics on strongly pseudoconvex domains in $\mathbb{C}^{n}$ with smooth boundary, Trans. Amer. Math. Soc. 207 (1975), 219-240.

[G2] _ Distortion theorems for holomorphic maps between convex domains in $\mathbb{C}^{n}$, Complex Variables Theory Appl. 15 (1990), 37-42.

[G3] - Sharp constants for the Koebe theorem and for estimates of intrinsic metrics on convex domains, Proc. Sympos. Pure Math., vol. 52, part 2, Amer. Math. Soc., Providence, RI, 1991, pp. 233-238.

[H] G. M. Henkin, An analytic polyhedron is not biholomorphically equivalent to a strictly pseudoconvex domain, Soviet Math. Dokl. 14 (1973), 858-862.

[K] S. Kobayashi, Intrinsic distances, measures and geometric function theory, Bull. Amer. Math. Soc. 82 (1976), 357-416.

[Kr1] S. G. Krantz, Optimal Lipschitz and $L^{p}$ regularity for the equation $\bar{\partial} u=f$ on strongly pseudo-convex domains, Math. Ann. 219 (1976), 233-260.

[Kr2] _ Function theory of several complex variables, Wiley, New York, 1982.

[LG] P. Lelong and L. Gruman, Entire functions of several complex variables, Grundlehren Math. Wiss., vol. 282, Springer-Verlag, Berlin, 1986.

[L] L. Lempert, La métrique de Kobayashi et la répresentation des domains sur la boule, Bull. Soc. Math. France 109 (1981), 427-474.

[Me] P. R. Mercer, Complex geodesics and iterates of holomorphic maps on convex domains in $\mathbb{C}^{n}$, Trans. Amer. Math. Soc. (to appear).

[Mi1] K. Miller, Barriers on cones for uniformly elliptic operators, Ann. Mat. Pura Appl. 76 (1967), 93-105.

[Mi2] _ Extremal barriers on cones with Phragmen-Lindelöf theorems and other applications, Ann. Mat. Pura Appl. 90 (1971), 297-329.

[O] J. K. Oddson, On the boundary point principle for elliptic equations in the plane, Bull. Amer. Math. Soc. 74 (1968), 666-670.

[P] S. I. Pinčuk, On proper holomorphic mappings of strictly pseudoconvex domains, Siberian Math. J. 15 (1974), 644-649.

[R1] R. M. Range, On the topological extension to the boundary of biholomorphic maps in $\mathbb{C}^{n}$, Trans. Amer. Math. Soc. 216 (1976), 203-216.

[R2] The Caratheodory metric and holomorphic maps on a class of weakly pseudoconvex domains, Pacific J. Math. 78 (1978), 173-189.

[Roy] H. L. Royden, Remarks on the Kobayashi metric, Several Complex Variables. II (Maryland, 1970), Springer, Berlin, 1971, pp. 125-137.

[Ru] W. Rudin, Function theory in the unit ball of $\mathbb{C}^{n}$, Grundlehren Math. Wiss., vol. 241, Springer-Verlag, Berlin, 1980.

Department of Mathematics, University of Toronto, Toronto, Ontario, Canada M5S 1A1

Current address: Department of Mathematics, University of North Carolina, Chapel Hill, North Carolina 27599-3250

E-mail address: mercer@math.unc.edu 